scleral search coil technique ${ }^{4}$; I suspect that it would have shown a torsional component and that this patient also had jerk-waveform see-saw nystagmus.

Jerk-waveform see-saw nystagmus occurs with unilateral mesodiencephalic lesions, presumed due to selective unilateral inactivation of the torsional eye velocity integrator in the interstitial nucleus of $\mathrm{Cajal}^{2}$; during the fast (jerk) phases the upper poles of both eyes rotate toward the side of the lesion. With lateral medullary injury the fast phases of the torsional component jerk away from the side of the lesion. ${ }^{5}$ In both situations the torsional component is always conjugate. With mesodiencephalic lesions the vertical component is always disjunctive, but with medullary lesions it may be either conjugate (usually upward) or disjunctive. ${ }^{2}$

PATRICK LAVIN Departments of Neurology and Ophthalmology, Vanderbilt University Medical Center, 2100 Pierce Ave. Nashville, TN 37212, USA patrick.lavin@mcmail.vanderbilt.edu

1 Pieh C, Gottlob I. Arnold Chiari malformation and nystagmus of skew. $\mathcal{F}$ Neurol Neurosurg Psychiatry 2000;69:124-6.

2 Halmagyi GM, Aw ST, Dahaene I, et al. Jerk-waveform see-saw nystagmus due to unilateral meso-diencephalic lesion. Brain 1994 117:789-803.

3 Lavin PJM. Diplopia, nystagmus and other ocular oscillations. In: Daroff RB, Fenichel GM Marsden CD, et al, eds. Neurology in clinical practice. Boston: Butterworth, 2000;16:203-27.

4 Collwejin H, Vaan der Steen J, Ferman L, et al. Human ocular counterroll: assessment of static and dynamic properties from electromagnetic search coil readings. Exp Brain Res 1985;59: 185-1196.

5 Morrow MJ, Sharpe JA. Torsional nystagmus in the lateral medullary syndrome. Ann Neurol 1988;24:390-8.

\section{The authors reply:}

We thank Lavin for his interesting comments.

We stated in our article that the possibility of a fine see-saw nystagmus could not be excluded. We did re-evaluate our patients with a torsional coil and did not record a torsional component. However, because of the fast improvement in both patients, all the eye movement abnormalities on re-evaluation were minimal. Clinically, even in the stage of maximal abnormalities, in one patient we did not detect any torsional component, which suggests that if there was an element of see-saw nystagmus, it was subclinical.

We did not state that the type of nystagmus associated with the Arnold-Chiari malformation was unique, precisely because we could not rule out with total certainty a see-saw nystagmus, which has been reported in one patient with the malformation. We did, however, point out that this association is unusual.

Because of the lack of strong evidence of a torsional component to the dissociated vertical nystagmus, we preferred the term, kindly suggested by a reviewer, "nystagmus of skew". This would represent a more inclusive, descriptive term, of which both the pendular and the jerk see-saw nystagmus forms and the dissociated vertical nystagmus without demonstrable torsional component would represent subvariants.

$\mathrm{C}$ PIEH

Kantonsspital St Gallen,

Switzerland

I GOTTLOB

Department of Ophthalmology, Leicester Royal Infirmary,

Leicester LE1 5WW, UK
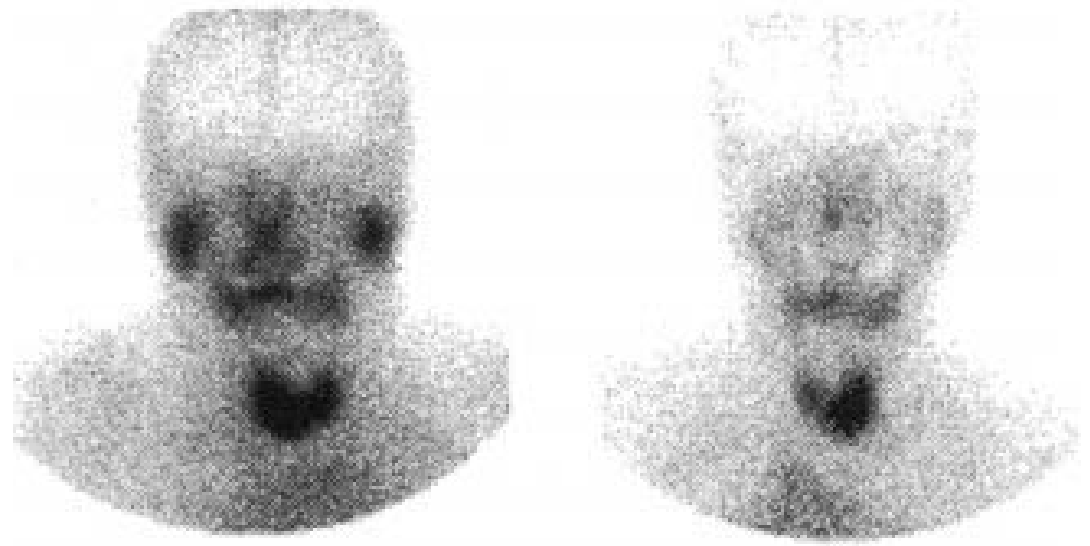

Figure 1 Scintigraphy of the head of patient 1 showing technetium $99 \mathrm{~m}$ uptake one day before (left) and 7 days after (right) retrograde transductal BoNT/A injection into the salivary glands, showing significant reduction of tracer uptake after the BoNT/A injection (quantification: parotid gland left $-59 \%$, right $-48 \%$; sublingual gland left $-10 \%$; right $-56 \%$ ).

Botulinum toxin for the treatment of sialorrhoea in ALS: serious side effects of a transductal approach

We have read with interest the article by Giess et $a l,{ }^{1}$ which showed that botulinum toxin A (BoNT/A) might be a new treatment option for sialorrhoea in patients with bulbar palsy. We have recently conducted a similar study which was interrupted due to serious side effects.

In September 1998 we injected $25 \mathrm{MU}$ Botox into the parotid glands of a 59 year old women who had ALS with pronounced bulbar palsy. We noticed a reduction of the sialorrhoea but facial weakness on the left side worsened significantly.

After this experience we developed a protocol for the treatment of sialorrhoea in patients with ALS with bulbar palsy by retrograde injection of BoNT/A through the salivary duct into the salivary glands. We chose the retrograde way of administration of BoNT/A for this pilot study because we thought that this technique would avoid facial weakness.

After informed consent the patients received 12.5 mouse units (MU) BoNT/A $\left(\right.$ Botox $^{\mathrm{R}}$ ) retrogradly into each parotid and sublingual gland from a small catheter inserted into the salivary duct. Neurological examination and quantification of saliva production were performed before the BoNT/A injection and on days $1,3,7,14$, and 28 , as well as after 2 and 3 months. Technetium $99 \mathrm{~m}$ scintigraphy was performed before and 7 days after the injection. Quantification of saliva production was performed with a simple method: the patients were asked to expectorate as much saliva as possible into a paper handkerchief for 10 minutes. This procedure was repeated twice. The mean of the difference in weight of the handkerchief before and after these procedures was taken as the maximum expectorated saliva production (MESP). Quality of life and the clinical effect of the treatment were evaluated by a questionnaire.

We treated a 60 year old woman (patient 1) and a 69 year old women (patient 2) with this new technique. Both had certain ALS according to the El Escorial criteria, with severe bulbar palsy with durations of 23 and 28 months respectively. Both patients had a significant reduction of MESP seven days after the injection ( $76 \%$ and $58 \%$; from 5420 $\mathrm{mg}$ to $1301 \mathrm{mg}$, and 4365 to $1829 \mathrm{mg}$ ) which lasted for 4 to 8 weeks. Technecium $99 \mathrm{~m}$ scintigraphy showed a significant reduction of radiotracer uptake into the injected salivary glands in both patients (figure). Both patients estimated the injection procedure as painful. Patient 1 developed a severe swelling of the right sublingual salivary gland and base of the tongue 3 days after the injection which was treated with antibiotics and corticosteroids. Patient 2, who was able to swallow with difficulty before the injection, mentioned impairment of swallowing between days 4 and 21. Both patients had a "moderate" improvement of sialorrhoea but did not want the injections to be repeated. After these experiences we decided to stop the pilot study.

The injection of BoNT/A through the salivary duct reduces the activity of the salivary glands significantly for several weeks but has serious side effects. Local and systemic effects of BoNT/A are probably pronounced in ALS. $^{2}$ Subclinical EMG abnormalities distant to the injection sites have been described in therapeutic doses, but also systemic weakness has been found. ${ }^{3}$ As there are some reports that BoNT/A injections, even in low doses, may exaggerate pre-existing neuromuscular diseases, ${ }^{24}$ careful monitoring of neurological symptoms, which is difficult in a progressive disease, is needed to exclude side effects of BoNT/A. The drug is effective in reducing drooling ${ }^{5}$ but we need more data about the safety of BoNT/A before it can be used safely for the treatment of sialorrhoea in ALS. The transductal approach in particular seems to have unacceptable side effects.

M G M WINTERHOLLER F J ERBGUTH Department of Neurology,

Friedrich-Alexander-Universität Erlangen, Schwabachanlage 6, D-91054 Erlangen, Germany $S$ WOLF

Department of Otorhinolaryngology S KAT

Department of Nuclear Medicine

Correspondence to: Dr M GM Winterholler, MD wiho.erlangen@t-online.de 
1 Giess R, Naumann M, Werner E, et al. Injections of botulinum toxin $\mathrm{A}$ into the salivary glands improve sialorrhoea in amyotrophic lateral sclerosis. $\mathcal{F}$ Neurol Neurochir Psy chiatry 2000;69:121-123.

2 Mezaki T, Kaji R, Kohara N, et al. Development of general weakness in a patient with amyotrophic lateral sclerosis after focal botulinum toxin injection. Neurology 1996;46: 845-6.

3 Bakheit AM, Ward CD, McLellanDL. Generalized botulism-like syndrome after intramuscular injections of botulinum toxin type A. A report of two cases. $\mathcal{F}$ Neurol Neurochir Psychia try 1997;62:196-200.

4 Erbguth F, Claus D, Engelhardt A, et al. Systemic effect of local botulinum toxin injections unmasks subclinical Lambert-Eaton myasthenic syndrome. 7 Neurol Neurochir Psychiatry 1993;56:1235-36.

5 Jost WH. Treatment of drooling in Parkinson's disease with botulinum toxin. Mov Disord 1999;14:1057.

\section{The authors reply:}

We appreciate the comments by Winterholler et al on our article ${ }^{1}$ on botulinum toxin (BTX/A) treatment of sialorrhoea in patients with amyotrophic lateral sclerosis (ALS). Although we did not find any serious side effects after transcutaneous injections of BTX/A into the parotid and submandibular glands Winterholler et al report on sublingual salivary gland infection in one patien and deterioration of dysphagia in another patient after a transductal approach. These complications support our notion that the individually tolerated dose of BTX/A in patients with ALS may be low and also indicate that the transcutaneous approach as performed in several studies ${ }^{1-3}$ may be safer than the retrograde transductal injection. This is not unexpected as the transductal approach has possibly a higher risk of infection because of the reduced salivary gland secretion rate found in patients with ALS. ${ }^{1}$ In addition, the total dose of $25 \mathrm{MU}$ Botox for the sublingual glands may be rather high in view of the close anatomical relation of these glands to the pharyngeal muscles. We therefore underscore our previous suggestion to start with injections of the parotid glands alone and to cautiously escalate the dose and number of injected glands. ${ }^{1}$ In view of the potential risk of BTX/A in deteriorating ALS symptoms injections should be restricted to otherwise intractable and extremely disabled patients with ALS who have sialorrhoea.

M NAUMANN R GIESS

K SCHWAGER

K V TOYKA

Department of Neurology and Department of Otorhinolaryngology,

Bayerische-fulius-Maximilians-Universität, fosef-Schneider-Strasse 11, 97080 Würzburg, Germany

Correspondence to: Dr M Naumann naumann@mail.uni-wuerzburg.de

1 Giess R, Naumann M, Werner E, et al. Injections of botulinum toxin $A$ into the salivary glands improve sialorrhoea in amyotrophic lateral sclerosis. $\mathcal{F}$ Neurol Neurosurg Psychiatry 2000;69:121-3.

2 Jost WH. Treatment of drooling in Parkinson's disease with botulinum toxin. Mov Disord 1999;14:1057.

3 Bhatia KP, Münchau A, Brown P. Botulinum toxin is a useful treatment in excessive drooling of saliva [letter]. I Neurol Neurosurg Psychiatry 1999;67:679.
Treatment of early onset Parkinson's disease with ropinirole

The recent editorial ${ }^{1}$ supporting initial treatment of early onset Parkinson's disease with a dopamine agonist hinged in part on the demonstration $^{2}$ in 268 patients that treatment of early onset Parkinson's disease with ropinirole alone or with supplementary levodopa/dopa decarboxylase inhibitor (benserazide) (LD/DDI) resulted in substantially less dyskinesia than with LD/DDI alone, with only slightly less motor benefit. Five per cent of patients on ropinirole alone developed dyskinesia after 5 years, compared with $25 \%$ with ropinirole plus LD/DDI, and $45 \%$ of those on LD/DDI alone). The trial design allowed LD/DDI supplementation if response was inadequate and additional trial drug could not be tolerated. Up to $24 \mathrm{mg}$ ropinirole and $1200 \mathrm{mg} \mathrm{LD} / \mathrm{DDI}$ daily were allowed. Sixty six per cent of patients completing the ropinirole arm required supplementation, the average mean daily dose of ropinirole at 5 years being $16.5 \mathrm{mg}$, compared with $753 \mathrm{mg}$ of LD/DDI when the second was used alone.

It is unfortunate that the study required a three times daily dosage regime. It seems possible that this accounts for the surprising $33 \%$ of patients on LD/DDI alone who withdrew as a result of early adverse events, and for the occurrence of nausea in $49.4 \%$ of patients on LD/DDI alone. Whether smaller, more frequent, dosage would have allowed better tolerance of and motor response to ropinirole, it is not surprising that frequent dyskinesia was seen at 5 years on three times daily dosage of LD/DDI. A substantial proportion of patients on LD/DDI (43.8\%) were also on selegiline, amplifying the effect substantially. By comparison, the 5 year study of immediate release (IR) and controlled release (CR) LD/DDI (carbidopa) in 681 patients, ironically reported earlier in 1997, and later in $1999,{ }^{3}$ in whom dosage could be adjusted up to five or more times a day resulted not only in a lower frequency of dyskinesia $(20.6 \% \mathrm{IR}, 21.7 \% \mathrm{CR})$ at 5 years but also a lower mean total daily dose $(426 \mathrm{mg}$ IR; 510 mg CR (bioequivalent)).

Whereas it may be argued that different drug preparations and methods of assessment invalidate comparison, it may be simply that less frequent higher pulsatile dosage provokes not only greater peak dose dyskinesia but also, as a mirroring effect, greater off time as postsynaptic mechanisms adapt to cope with surges of dopamine and perhaps lose sensitivity to troughs. Patients seen during troughs would be liable to have their dose increased. If the interdose interval were fixed this would lead to a vicious circle.

Given reports of long term resolution of dyskinesia and on/off effects in response to various methods of continuous stimulation, at an appropriate strength, including continuous daytime jejunal infusion of LD/DDI (with little or no change in $\mathrm{LD} / \mathrm{DDI}$ dosage requirement over 57 months), ${ }^{4}$ and of a neuroprotective effect of levodopa, ${ }^{5}$ the results of Rascol et $a l^{2}$ should not dissuade others from pursuing oral treatment with LD/DDI in a more frequent, lower dose regime. With gradual (allowing for the long duration action of levodopa) titration of slow release LD/DDI dosage and interdose interval (if necessary using a timer), against response and compliance of patient (or carer), it may in theory and, with sufficient observation and titration, in practice be possible to approximate to such a steady state stimulation and response. This would have potentially less risk for developing hallucinations, and would cost less.

J R PONSFORD

Department of Neurology, Walsgrave Hospital NHS Trust, Clifford Bridge Road, Coventry CV2 2DX, UK

1 Brooks DJ. Dopamine agonists: Their role in the treatment of Parkinson's disease [editorial]. $\mathcal{F}$ Neurol Neurosurg Psychiatry 2000;68:685-90.

Neurol Neurosurg Psychiatry 2000;68:685-90.
Rascol O, Brooks DJ, Korczyn AD, et al. A five year study of the incidence of dyskinesia in year study of the incidence of dyskinesia in
patients with early Parkinson's disease who patients with early Parkinson's disease who
were treated with ropinirole or levodopa. $N$ were treated with ropinirole
Engl f Med 2000;342:1484-91.

3 Koller WC, Hulton JT, Tolosa E, et al. Immediate release and controlled release carbidopa/ levodopa in Parkinson's disease. Neurology 1999;53:1012-19.

4 Syed N, Murphy J, Zimmermann T Jr, et al. Ten years experience with enteral levodopa infusions for motor fluctuations in Parkinson's disease. Mov Disord 1998;13:336-8.

5 Murer G, Dziewczapolski G, Menalled LB, et al. Chronic levodopa is not toxic for remaining dopamine neurons, but instead promotes their dopamine neurons, but instead promotes their lesions. Ann Neurol 1998;43:561-75.

Brooks replies:

Ponsford seems to focus primarily on the design and findings of the 056 trial of ropinirole versus levodopa in early Parkinson's disease recently reported in the $N$ Engl F $\mathrm{Med}^{1}$ rather than the editorial as a whole; however, to take up his points:

Firstly, he suggests that it is unfortunate that the 056 trial required a three times daily levodopa dosage regime as use of more frequent smaller doses could have reduced the incidence of dyskinesias. We chose a three times daily regime in part to match the three times daily regime of ropinirole and also because it was thought that this regime reflected common clinical practice in patients with early Parkinson's disease. A trial formally comparing use of multiple low doses of levodopa versus a three times daily medium dose regime in early Parkinson's disease would, however, be of great interest. It might well be that the multiple low dose approach in early disease would spare complications but this has yet to be shown. Addition of a catechol-O-methyltransferase inhibitor to smooth out the plasma levodopa profile in early Parkinson's disease might also prove beneficial.

Secondly, he suggests that the allowed presence of selegeline may have magnified the tendency of levodopa to cause complications. This could indeed be the case although stratifying for selegeline usage in the levodopa arm did not highlight any such effect.

Thirdly, he suggests that allowing the use of slow release levodopa preparations in early Parkinson's disease could have been beneficial. There is currently no trial data to support this viewpoint; on the contrary, early use of either slow release madopar or sinemet has been reported to be associated with a similar prevalence of complications as the use of standard preparations.

My current feeling is that early use of dopamine agonists in suitable patients with Parkinson's disease remains a reasonable strategy to delay complications. It may well be that in the future, however, a more optimal way of delivering levodopa is devised which achieves continuous and uniform dopaminergic stimulation and so reduces the prevalence of fluctuations and dyskinesias.

D J BROOKS Neurology Department, Imperial College School of Medicine, Hammersnith Hospital, London W12 ONN, UK 